\title{
Miscible Blend Dynamics and the Length Scale of Local Compositions
}

\author{
VICTORIA GARCÍA SAKAI, ${ }^{1}$ JANNA K. MARANAS, ${ }^{1}$ ZEMA CHOWDHURI, $^{2}$ INMACULADA PERAL, ${ }^{2}$ \\ JOHN R. D. COPLEY ${ }^{2}$ \\ ${ }^{1}$ Department of Chemical Engineering, The Pennsylvania State University, University Park, Pennsylvania 16802 \\ ${ }^{2}$ NIST Center for Neutron Research, National Institute of Standards and Technology, Gaithersburg, Maryland 20899-8562
}

Received 8 November 2004; revised 18 April 2005; accepted 22 June 2005

DOI: 10.1002 / polb.20562

Published online in Wiley InterScience (www.interscience.wiley.com).

\begin{abstract}
We consider the influence of a local, or effective, composition on dynamics in the miscible polymer blend PEO/PMMA. Quasielastic neutron scattering in combination with deuterium labeling is employed to determine characteristic relaxation times of the PEO component over spatial scales from 3 to $10 \AA$. Information about the distribution of relaxation times is obtained indirectly from the stretching parameters in a stretched exponential fit. We examine the behavior of these parameters with spatial scale and temperature, finding that their variation supports a distribution of PEO mobility in the blend which is far wider than pure PEO and narrows with decreasing temperature for small spatial scales. This is linked to the concept of local compositions defined over varying spatial scales, and indicates that the concept of a local composition, linked to PEO dynamics, is important in this system. ( 2005 Wiley Periodicals, Inc. J Polym Sci Part B: Polym Phys 43: 2914-2923, 2005
\end{abstract}

Keywords: neutron scattering; polymer blends; dynamics

\section{INTRODUCTION}

Shifts in component dynamics in miscible polymer blends arise from an effective local composition, $\phi_{\text {eff, }}$ which differs from the bulk. One proposal for the origin of this local composition is chain connectivity, ${ }^{1}$ in which $\phi_{\text {eff }}$ is enhanced in one component on a length scale comparable to the Kuhn length. This approach can explain many observations common to miscible blends: these include the broadening of calorimetric $T_{\mathrm{g}}$, shifts from pure component dynamic properties that are smaller for the low $T_{\mathrm{g}}$ component, and failure of time-temperature superposition. The

Correspondence to: Janna K. Maranas (E-mail: jmaranas@ psu.edu)

Journal of Polymer Science: Part B: Polymer Physics, Vol. 43, 2914-2923 (2005) (ㅇ) 2005 Wiley Periodicals, Inc. chain connectivity picture has recently been tested on a large number of miscible blends where data is available, ${ }^{2}$ and in many cases is able to successfully correlate the data.

Another suggested origin ${ }^{3-5}$ is that fluctuations in concentration lead to a distribution of local compositions on length scales (cooperative volumes) similar to the Donth model for the glass transition. The effect of concentration fluctuations on dynamics should be most prominent near a phase boundary, leading to a large correlation length $\xi$, and when the glass transition temperatures of the two components are well separated. In this case, fluctuations in local composition occurring over large length scales can lead to a bimodal distribution of relaxation times of the low- $T_{\mathrm{g}}$ component. The probability for mobility of a local region depends on local composition and the cooperative volume required for 
mobility at that composition. Longer relaxation times arise from regions of local composition close to the bulk; these are very probable but have large cooperative volumes. Shorter relaxation times result from local compositions rich in the low- $T_{\mathrm{g}}$ component; this is a smaller probability event but one that requires far smaller cooperative volumes for mobility. Measurements with sufficient dynamic range detect both relaxations-each connected to a local composition defining a local $T_{\mathrm{g}}$ and cooperative volume.

Both of the models described above are based on a locally defined composition distinct from that of the bulk. The concentration fluctuation model also requires a size-dependent distribution of local compositions. Quasi-elastic neutron scattering (QENS) presents a unique opportunity to manipulate the spatial scale over which the measurement is made, and thus observe spatially resolved dynamics. Because the local composition is a function of the volume used to define it, varying spatial scale over the appropriate range can be expected to probe regions of different local, or effective, compositions. Incoherent QENS experiments average self motion of protons, which dominate the scattering, over subvolumes with spatial scales that can be systematically varied from the order of chemical bonds through the intermolecular packing region (as defined by the first peak in $S(Q)$ ) to the Kuhn length. The spatial scale is set by the momentum transfer $Q$, which is inversely related to distance: $Q=2 \pi / r$. At the higher end of this $Q$-range, intramolecular bonding strongly biases the effective concentrationonly the closest possible interchain contacts will contribute. As the spatial scale increases, more neighboring chains enter the observation window and local intermolecular packing influences the effective concentration. At the high end of the spatial range, the intermolecular pair distribution function approaches unity, indicating that local packing variations are no longer important. This is expected to occur near the Kuhn length $b$. At this point, the effective concentration should approach the bulk value, unless concentration fluctuations are significant over large spatial scales (i.e., $\xi>b$ ). Varying spatial scale over this range is also expected to affect the distribution of local compositions: a smaller range is expected where intramolecular bonding dominates the local composition than where intermolecular packing is important.

Average relaxation times extracted from the spectra depend on the spatial scale, and often mechanisms of motion can be deduced from this dependence. For example, in simple continuous diffusion the relaxation times will have a $Q^{2}$ dependence. The distribution of relaxation times present in a given sample also may be characterized, although indirectly, as it appears as a stretching of the spectra in the time domain (i.e., the spectra have a different shape), or a failure of a single Lorentzian to fit the data in the frequency domain. This distribution of relaxation times may be connected to the distribution of local compositions: fast moving protons may be surrounded by a local environment rich in the more mobile component. It is also possible that the two distributions are unrelated. We propose that this question may be answered by considering variations in the shape of the spectra, with spatial scale.

There are two possibilities, the first being that a distribution of relaxation times exists in a miscible blend because of heterogeneous dynamics, as in pure polymers. In this case, each proton belongs to a dynamic (for example, fast or slow) region, which is likely to be of a size scale (30$100 \mathrm{~nm}$ ) larger than the spatial range addressed in QENS measurements. Since the mobility of each proton is set by the dynamic region to which it belongs, and is thus independent of local composition, the distribution of relaxation times should not be a function of spatial scale, as is the case for pure polymers. In the second possibility, the distribution of proton mobilities arises because of a distribution of local compositions, unique to blends. In this case, changing the spatial scale of the measurement will affect this distribution, as described above. In particular, focusing the spatial scale to a size small enough (that the protons rarely sample the environment outside their own chain) should narrow the distribution of relaxation times, and in the limiting case, the distribution should approach that of the pure component. As a result, a narrowing distribution with decreasing spatial scale would indicate that local compositions influence $\mathrm{dy}$ namic behavior, as this result is not expected otherwise.

The chain connectivity model connects dynamics with a particular length scale: the Kuhn length. In this case, average dynamic properties, such as the relaxation time, are controlled by the effective composition defined by $b$. Our purpose here is different. We do not address average relaxation times, but rather their distribution. The concept of a distribution of local 
compositions is not required for the chain connectivity picture, nor is it precluded. On the other hand, the existence of such a distribution is an integral part of the concentration fluctuation theory.

We have chosen a system for our measurements that should be an ideal candidate for concentration fluctuations: the low $T_{\mathrm{g}}$ component in a miscible blend with large $\Delta T_{\mathrm{g}}$ (poly(ethylene oxide) PEO in blends with poly(methyl methacrylate) (PMMA). In addition to the features just mentioned, PEO/PMMA is one system where the chain connectivity picture cannot correlate all available data. ${ }^{2,6}$ These data at very low PEO concentrations (3\%) show little difference from those at higher concentrations (up to 30\%). This would indicate that concentration fluctuations are unimportant. Clearly, the controlling factors in dynamics of this blend are currently unknown. If concentration fluctuations influence PEO dynamics in blends with PMMA, it is unlikely that QENS could directly capture the resulting bimodal relaxation, since the spatial range $(\sim 2.5-15 \AA$, depending on spectrometer specifications) is not large enough to probe cooperative volumes rich in PMMA, and relaxation times of such regions would be far longer than the timescales of QENS instruments (0.01 ps-10 ns).

\section{EXPERIMENTAL}

Blends containing 10, 20, and 30\% hydrogenated PEO and deuterated PMMA were prepared as described in a previous publication. ${ }^{7}$ Dynamic measurements were performed using the diskchopper time-of-flight spectrometer ${ }^{8}$ (DCS) and the high flux backscattering spectrometer (HFBS $)^{9}$ at the NIST Center for Neutron Research in Gaithersburg, MD, USA. The DCS was operated at an incident wavelength of $4.2 \AA$ and an energy resolution of $81.6 \mu \mathrm{eV}$. A dynamic range of \pm 20 $\mu \mathrm{eV}$ was used for the HFBS, with an energy resolution of $0.87 \mu \mathrm{eV}$. Temperatures between $T=308$ $\mathrm{K}$ and $T=440 \mathrm{~K}$ were measured-a range that includes the blend $T_{\mathrm{g}}(348 \mathrm{~K})$ and the $T_{\mathrm{g}}$ of pure PMMA (391 K). The measured neutron intensities were corrected using software developed by NIST $^{10}$ (DAVE) for detector efficiency (comparison with a Vanadium standard), for scattering from the empty annular can and for time independent background scattering. The raw spectra were Fourier transformed into the time domain ${ }^{7}$ where the instrument resolution was removed.

\section{RESULTS AND DISCUSSION}

Intermediate scattering functions of $\mathrm{PEO}$ alone and in blends with PMMA are given in Figure 1. At first glance, it appears that a bimodal relaxation is observed: two dynamic regimes are apparent at all compositions. We note that the process does not decay to zero for the blends, leaving open the possibility of another relaxation at longer times. With further examination, pure PEO also shows two relaxations and thus we observe only one contribution (if two exist) to the structural $\alpha$-relaxation of PEO in the blends. This initial fast decay has been observed in other pure polymers (polyisoprene,${ }^{11}$ polybutadiene, ${ }^{11}$ polyisobutylene, ${ }^{12}$ and several polyolefins ${ }^{13,14}$ ), although it has not previously been investigated in blends.
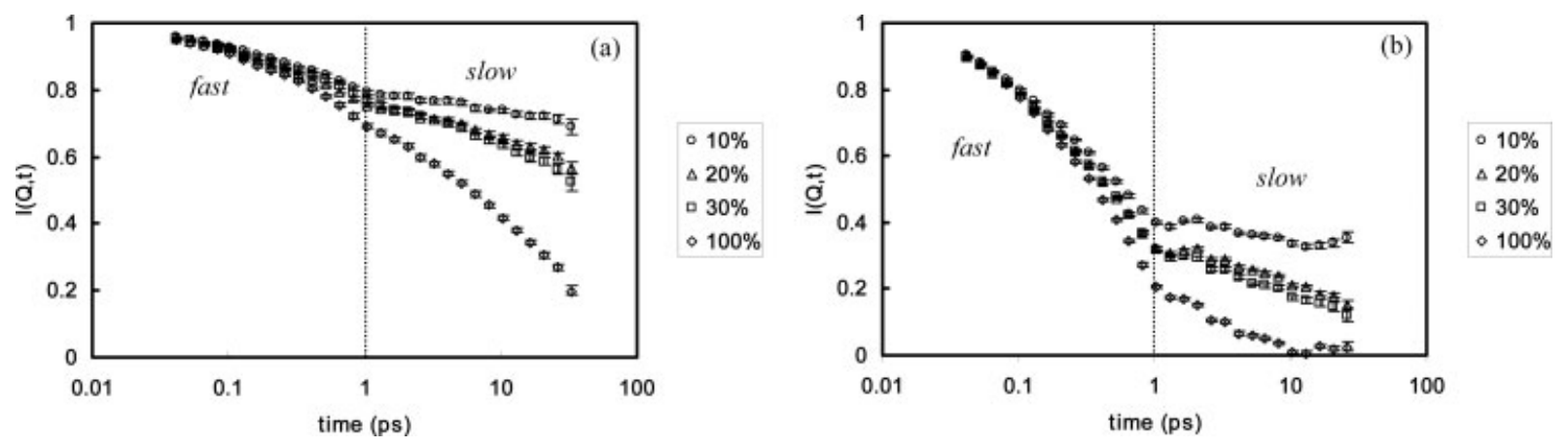

Figure 1. Effect of composition on the self intermediate scattering function of PEO in blends with PMMA at (a) $Q=0.89 \AA^{-1}$ and (b) $Q=2.51 \AA^{-1}$. The temperatures of the measurements are $343 \mathrm{~K}$ (100\% PEO), $345 \mathrm{~K}$ (10 and 30\% PEO) and $348 \mathrm{~K}(20 \% \mathrm{PEO})$. 
To characterize the motion resulting in these spectra, it is useful to employ a fitting procedure that will return an average relaxation time and a parameter that characterizes the width of the distribution of relaxation times. We use the empirical Kolrausch-Williams-Watts (KWW) expression for this purpose.

$$
I(Q, t)=A(Q, T) \exp \left[-\left(\frac{t}{\tau(Q, T)}\right)^{\beta(Q, T)}\right]
$$

It is apparent from the spectra that the relaxations are very stretched, which presents a problem when fitting to an empirical function such as KWW. In particular, the stretching parameter, $\beta$, can be rather difficult to assign when the decay is small. We address this problem in two ways: extending the timescale by measuring the $20 \%$ sample on HFBS, which has higher resolution and thus reaches longer times, at four of the seven temperatures measured on DCS, and an error analysis procedure that returns the maximum and minimum values of parameters ( $\tau$ and $\beta$ ) providing a reasonable fit to the data (regardless of the values of the other parameters) as an error bar. The error analysis procedure is described in detail by García Sakai. ${ }^{7}$ In brief, a large number of data sets are synthesized from the intermediate scattering function and its associated errors. These data sets are fit, and the resulting parameters catalogued. The data points we report are the most probable result, while error bars represent the range of parameters returned in the fits. Parameter values outside of the error bars should be regarded as not providing a reasonable fit, regardless of the values of the remaining parameters. Note that in some cases, the decay is not complete enough to provide reasonable estimation of any parameters; in our fit procedure, this manifests itself as an extremely large error bar. Such a case is the slow decay for the $10 \%$ blend at $Q=2.51 \AA^{-1}$.

We verify that this procedure yields adequate results by testing the fit parameters obtained from the DCS data alone against the more complete decay provided by adding the HFBS data, where it is available. Figure 2 shows the combined data at $T=308 \mathrm{~K}$ for six momentum transfers. The lowest temperature is selected because the smallest decays are found for small $Q$ and low $T$, so it represents the most stringent test. The fits shown in Figure 2 are within the error bars returned from fitting the DCS data, but are

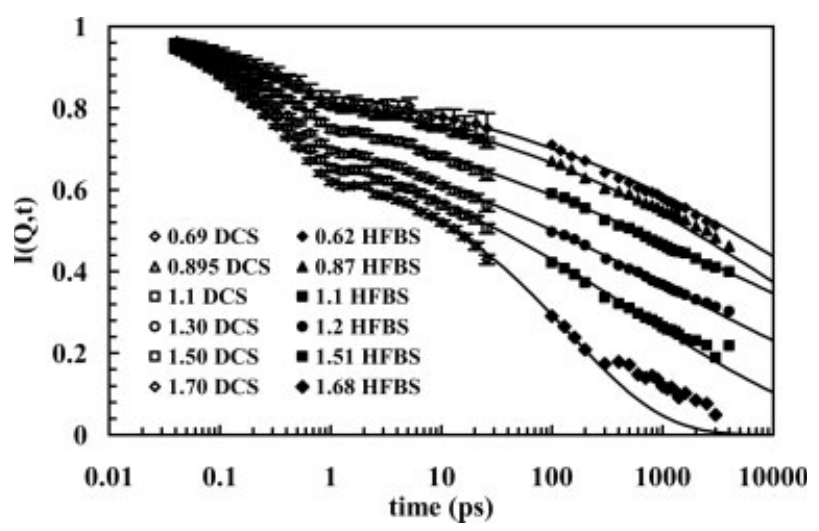

Figure 2. Combined DCS and HFBS scattering decay curves for hPEO in dPMMA at $308 \mathrm{~K}$. Seven spatial scales are shown. Lines represent a KWW fit with parameters falling within the error bars determined using the DCS data alone.

not always the most probable result. This analysis supports the fit procedure, indicating that its application where the HFBS data is not available is reasonable.

In Figure 3, we plot the relaxation times and error bars obtained from the fitting procedure for $Q=1.3 \AA^{-1}$ as a function of inverse temperature. Also provided are relaxation times for PEO in the PEO/PMMA blend from recent NMR results. ${ }^{6}$ It is clear that the slow process is comparable with the NMR results, both in temperature dependence and magnitude of relaxation times, while the fast process is considerably faster and has a weaker temperature dependence. Agreement between QENS and NMR for the 10\% blend is less satisfactory: we return to this point below.

\section{The Influence of Local Compositions}

Earlier, we proposed that a connection between the distribution of relaxation times and the distribution of local compositions might be revealed by investigating the shape of the relaxation curves as a function of spatial scale, the control of which is a feature unique to neutron measurements. We now discuss this idea, as applied to PEO motion in the PEO/PMMA blend. We focus on the slow decay of the $20 \%$ blend, with full analysis of all compositions to follow in a subsequent publication. To quantify the ideas presented in the introduction, we consider the stretching parameter in a $\mathrm{KWW}^{15}$ fit $(\beta)$ and the associated average relaxation time $(\tau)$. A de- 

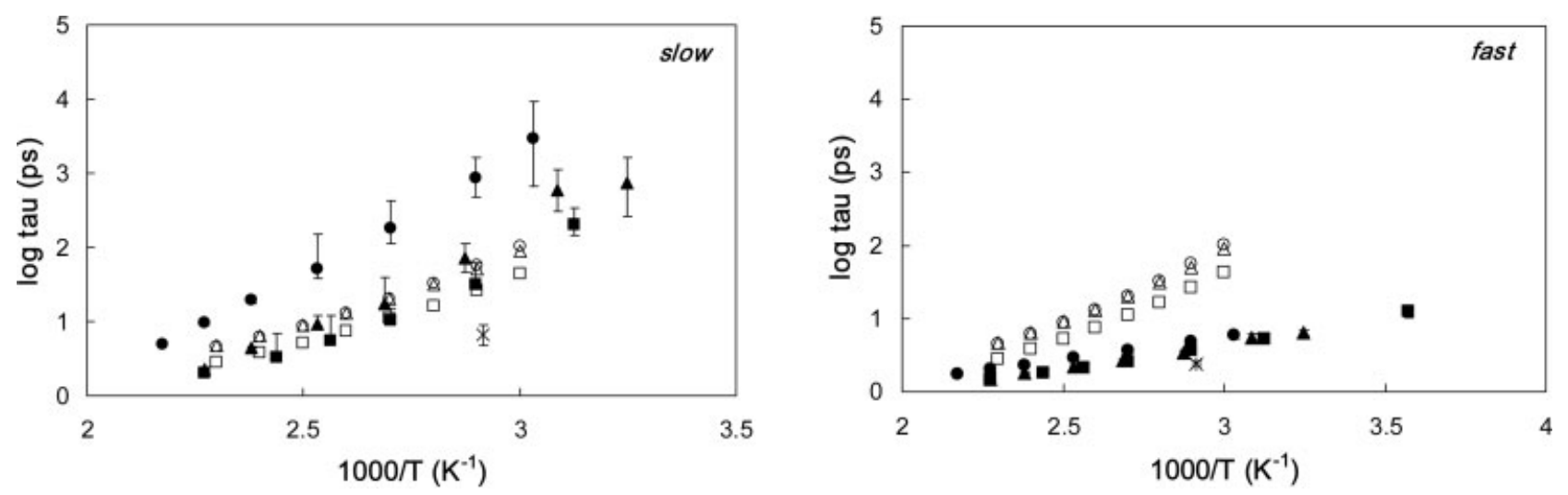

Figure 3. Relaxation times and associated error bars from QENS for the slow and fast processes of $\mathrm{PEO}$ in blends with PMMA. Also shown are data from NMR. ${ }^{6}$ Solid symbols: QENS relaxation times $Q=1.3 \AA^{-1}:(\bullet) 10 \%,(\mathbf{\Delta}) 20 \%,(\mathbf{\square}) 30 \%$, and $(\times) 100 \%$. Empty symbols: NMR segmental relaxation times: $(\bigcirc) 10 \%,(\triangle) 20 \%$, and ( $\square) 30 \%$.

crease in $\beta$ indicates a larger variety of individual relaxation times contributing to the average response, as can be shown mathematically. ${ }^{16}$ Note that this discards the possibility of a system of identically relaxing subvolumes, all of which are stretched in nature. ${ }^{17}$ We find this possibility to be small, given that the existence of dynamic heterogeneity has been observed both experimentally ${ }^{18}$ and with computer simulations. ${ }^{19}$

As described earlier, the measured spectra in a QENS experiment represent an average over all possible subvolumes of the prescribed size: as this size is manipulated by varying the momentum transfer, $Q$, both the average local composition of PEO segments, $\phi_{\mathrm{PEO}}$, and the distribution about this average, $P\left(\phi_{\mathrm{PEO}}\right)$, will vary. As a first approximation, the decay function reaches zero when the protons leave the subvolume specified by $Q$, and since they can leave in any direction, we regard the corresponding local composition as defined over a sphere of radius $r$, where $r=2 \pi / Q$. The largest length associated with this sphere is then $d=2 r=4 \pi / Q$. If the local compositions of PEO protons are unrelated to dynamic response, we expect to obtain parameter variations similar to pure PEO: the average relaxation times will decrease with decreasing spatial scale, but the shape of the spectra will not depend on $Q$. The latter is a consequence of the fact that a slow or fast moving PEO proton will contribute equally at any spatial scale, so the distribution of relaxation times-and therefore $\beta$-will remain the same. On the other hand, if the relaxation times of individual protons, $\tau_{\mathrm{i}}$, are linked to their local compositions, $\phi_{\mathrm{i}, \mathrm{PEO}}$, and both the average value
$\left(\phi_{\mathrm{PEO}}\right)$ and distribution $\left(P\left(\phi_{\mathrm{PEO}}\right)\right)$ of these compositions changes as a function of spatial scale, the parameters will vary differently. Specifically, $\phi_{\mathrm{PEO}}$ will increase and $P\left(\phi_{\mathrm{PEO}}\right)$ will narrow with decreasing spatial scale. The first of these would result in a decrease in average relaxation times; this will occur even if local composition and dynamics are unrelated, but if they are correlated, a change in $Q$ scaling might be expected. The narrowing of $P\left(\phi_{\mathrm{PEO}}\right)$ implies that $\beta$ will become larger with decreasing spatial scale.

Before presenting our results for PEO, we note that for the high $T_{\mathrm{g}}$ component in this blend, PMMA, ${ }^{7} \beta_{\text {blend }}$ is similar to $\beta_{\text {pure }}$ at all spatial scales. Its value is smallest near $T_{\mathrm{g}}$ and increases with temperature, leveling off at high temperature. This behavior is consistent with heterogeneous dynamics unrelated to $\phi_{\mathrm{PMMA}}$ and $P\left(\phi_{\text {PMMA }}\right)$.

Data for PEO support the local composition picture: results for $\beta_{\text {blend }}$ and $\beta_{\text {pure }}$ as a function of spatial scale are shown in Figure 4. The stretched nature of the decay in the blend data, clearly and outside of error, decreases as the spatial scale becomes smaller (larger $Q$ ). The largest spatial scales observable in our experiments define a local composition over $\sim 22 \AA$-these are small enough that $\phi_{\mathrm{PEO}}$ will be greater than the bulk composition, and that $P\left(\phi_{\mathrm{PEO}}\right)$ should be fairly wide. At these spatial scales, we find that the distribution of PEO mobility when blended with PMMA is much larger than in pure PEO. The smallest spatial scales observable in our experiments define a local composition over $\sim 5 \AA$, which includes both PEO-PEO and PEO- 


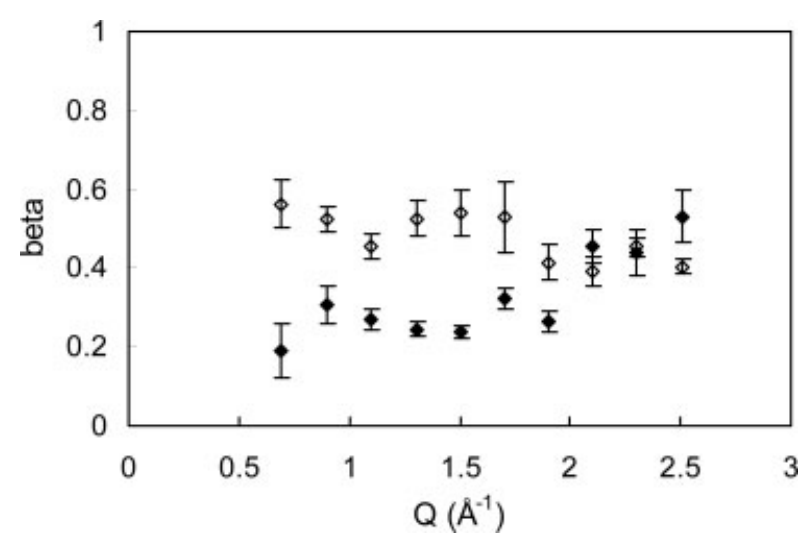

Figure 4. Spatial dependence of the stretching exponent $\beta$ for $(\diamond) 20 \% \mathrm{PEO}$ blend at $T=348 \mathrm{~K}$ and $(\diamond)$ pure $\mathrm{PEO}$ at $T=343 \mathrm{~K}$.

PMMA atomic spacings, and is not completely intramolecular in origin, as $S(Q)$ in this $Q$ range varies with composition. ${ }^{20}$ This means that a distribution of local compositions is still possible, but should be significantly narrower and closer to pure PEO than that expected over $22 \AA$. Here, $\beta_{\text {blend }} \cong \beta_{\text {pure, }}$ which indicates that the range of mobility of PEO protons in the blend is similar to that in pure PEO. The increase toward $\beta_{\text {pure }}$ begins after the $Q$ range of the first structure factor peak in PEO: $Q=1-2 \AA^{-1}$. $^{21}$ This is important with respect to PMMA, for which we do not observe a distribution of relaxation times broader than the pure component or a narrowing with decreasing spatial scale. The PMMA-labeled blend (hPMMA/dPEO) was measured on HFBS because of the slower motion of PMMA. Since this spectrometer has a maximum momentum transfer of $Q=1.7 \AA^{-1}$, considerably smaller than that of DCS, one could ask if the narrowing occurs, but we are unable to observe it. Since PMMA has a bulky side group, the intermolecular peak in $S(Q)$ is displaced to smaller $q$; in this case the peak is centered near $0.9 \AA^{-1}$ and ends near $1.2 \AA^{-1} .^{22}$ Thus in both cases, the range of our observations extends $\sim 0.5 \AA^{-1}$ beyond the intermolecular peak.

The temperature dependence of the stretching parameter is presented in Figure 5 for the largest and smallest spatial scales measured. At the smaller spatial scale $\left(Q=2.30 \AA^{-1}\right), \beta_{\text {blend }}$ is small at high temperatures, but increases rapidly below the $T_{\mathrm{g}}$ of PMMA, indicated in the figure by a dotted line. This unusual result suggests that the distribution of individual relaxation times narrows with decreasing temperature, in contrast to the usual observation that heterogeneity increases at lower temperatures. The reason for this is most likely that the PMMA rich side of $P\left(\phi_{\mathrm{PEO}}\right)$ becomes slower than the resolution of the spectrometer below the $T_{\mathrm{g}}$ of PMMA. These protons could contribute an elastic fraction, but would not influence the decay curves. This suggestion is supported by the temperature dependence of the average relaxation times, presented in Figure 6. The relaxation times for the largest spatial scale measured present an Arrhenius temperature dependence over the QENS time window, as expected and observed previously for this system. ${ }^{6}$ At small spatial scales and low temperatures, the relaxation times become independent of temperature, another unusual result, but one which is consistent with an increasing loss of contribution from the PMMA rich side of $P\left(\phi_{\mathrm{PEO}}\right)$.
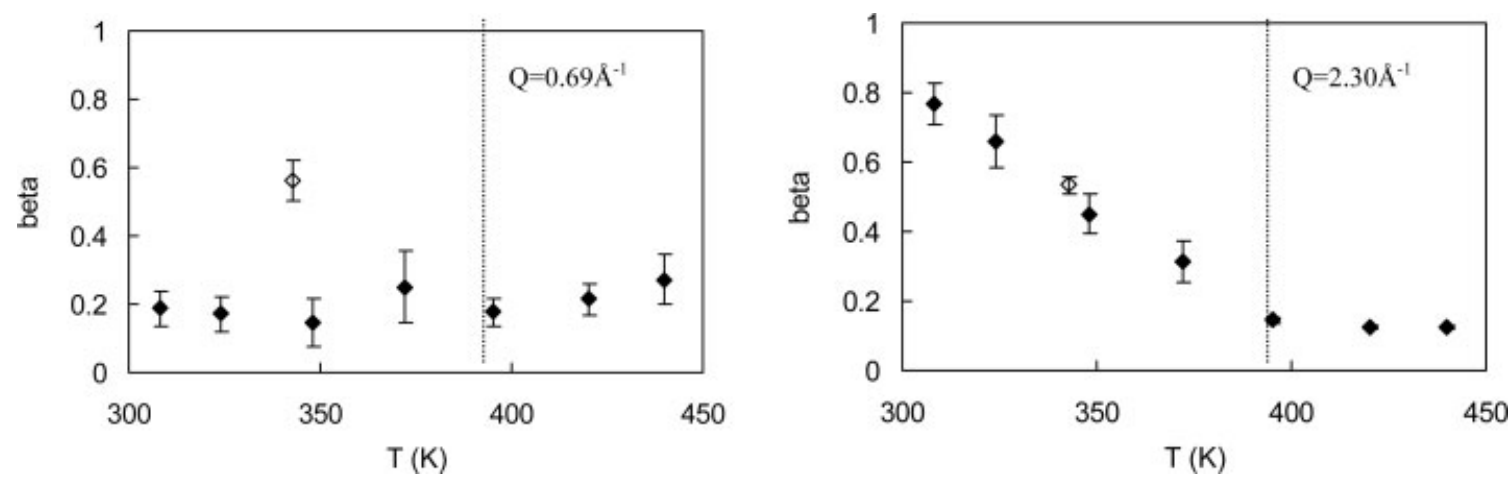

Figure 5. Effect of blending on the temperature dependence of the stretching exponent $\beta$. Results are shown at three spatial scales. $(\diamond) 20 \%$ PEO blend, $(\diamond)$ pure PEO. The dotted line shows the $T_{\mathrm{g}}$ of PMMA. 


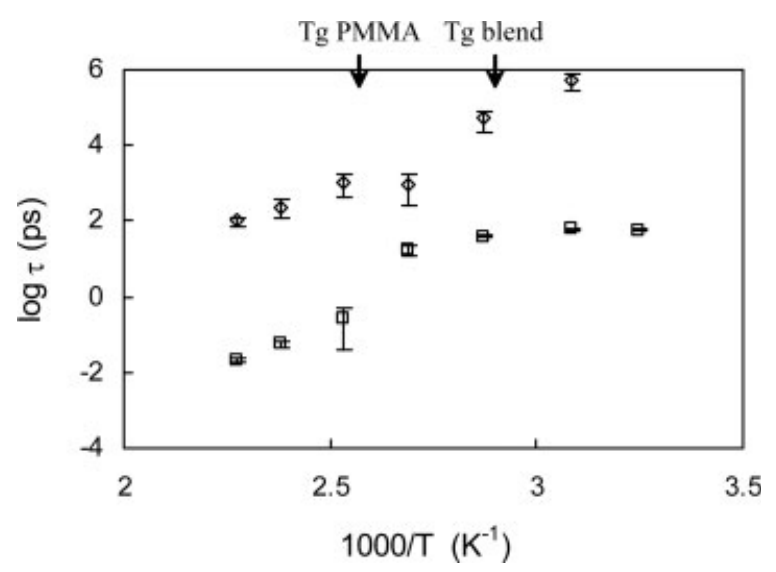

Figure 6. Temperature and spatial dependence of the average relaxation time $\tau$ of PEO in a $20 \%$ blend with PMMA: $(\diamond) Q=0.69 \AA^{-1}$ and $(\square) Q=2.30 \AA^{-1}$.

At the larger spatial scale $\left(Q=0.69 \AA^{-1}\right)$, $\beta_{\text {blend }}$ is temperature independent as well as being smaller than $\beta_{\text {pure. }}$. With a distribution of relaxation times resulting from dynamic heterogeneity (fast and slow moving regions of the sample unconnected to local compositions), $\beta$ will decrease as temperature is lowered. If instead the distribution of relaxation times is connected with a range of local compositions, it should only vary if this distribution is temperature dependent.

We first explore possible reasons for a narrowing of relaxation times at small spatial scales, other than that provided above. It is possible that the dynamics of PEO are less coupled to the dynamics of the surrounding environment because of lack of side groups, as suggested recently. ${ }^{2}$ In this case, we expect the distribution to narrow as the spatial scale is restricted because PEO is able to move unhindered if it does not encounter many surrounding PMMA segments. This effect, although plausible, would be expected to persist at high temperatures, in contrast to the current data. A narrowing of the relaxation time distribution could also result if all motion diffusive in nature is too slow for the instrument and a rotation, which for a glass would be a distribution of Lorentzians, ${ }^{23}$ emerges. However, the spatial dependence of relaxation times retains a diffusive character (power law in $Q$ ) at low temperatures for both PEO and the blends, as indicated in Figure 7, whereas rotational motion will either have relaxation times independent of $Q$ (two or three site jump models) or exhibit a distinct peak (four or more site jump models) ${ }^{24}$ Rotational motion is also an activated process, but relaxation times as the process narrows (large $Q$ and low $T$ ) are temperature independent. Finally, it is not clear what rotation would be observed in PEO, as it does not have side groups that could provide a clear rotation. Also shown in Figure 7 is the $Q$ dependence of relaxation times at high temperature, from which it is clear that at small spatial scales a second power law is observed with relaxation times that decrease with $Q$ more rapidly than the first. As mentioned above, this could occur if the effective concentration, $\phi_{\mathrm{PEO}}$, becomes richer in $\mathrm{PEO}$ as $Q$ increases.

A relaxation in addition to the $\alpha$ and $\beta$ processes, with a stretching parameter that approaches unity with decreasing temperature has been observed in pure PEO using dielectric spectroscopy. ${ }^{25}$ Jin et al. ${ }^{25}$ assign this process to a segmental motion and interpret it as occurring at the interface of crystalline and amorphous regions. It is also activated, with an activation energy of $-21 \mathrm{~kJ} / \mathrm{mol}$. This process has also been observed when PEO is blended with PMMA, although the stretching parameter is not available. ${ }^{26}$ In this case, it is ascribed to regions of PEO partial order that increase with aging. The timescale of the process, both in blends and in pure PEO is too slow for QENS, where one expects to observe only the region where all three processes are merged. It is possible that this process could be isolated at the appropriate spatial scale, but again this should persist at all temperatures, and the neutron data are not consistent with an activated process.

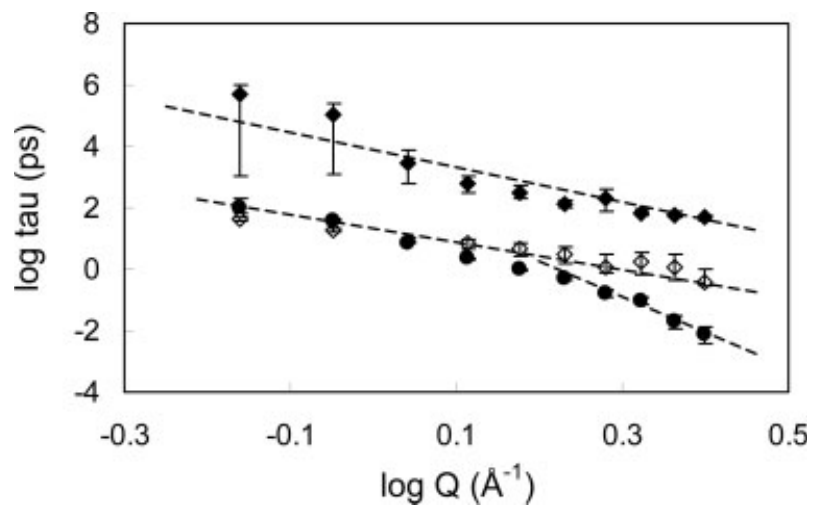

Figure 7. Spatial dependence of the characteristic relaxation times of $\mathrm{PEO}$ in a $20 \%$ blend with PMMA, ( ) at $T=324 \mathrm{~K}$ and $(\bullet)$ at $T=440 \mathrm{~K}$, and for pure $\mathrm{PEO}$ at $(\diamond) T=343 \mathrm{~K}$. 
As mentioned earlier, the broadening effect of local compositions begins to diminish at small spatial scales when the temperature is less than the $T_{\mathrm{g}}$ of PMMA. It is not immediately obvious why relaxation times do not also narrow at low temperature for the larger spatial scales. If the reason for the narrowing is that individual local compositions rich in PMMA become slower than the resolution of the spectrometer, this effect should also be observed at $0.69 \AA^{-1}$, as there the distribution will be centered around an $\phi_{\mathrm{PEO}}$ that is closer to the bulk composition than that at $2.3 \AA^{-1}$ and should have as many, if not more, PMMA rich regions. A possible answer to this is found in the timescale of motion as a function of spatial scale. The mobility of PEO is strongly $Q$ dependent, as can be seen in Figure 7. At high $Q$, motion can be four orders of magnitude greater than at large $Q$. As a result, the local environment surrounding a PEO proton may vary considerably over the characteristic time of motion at small $Q$, such that the response remains stretched. Over the characteristic times at large $Q$, which are far shorter, this is less likely to occur.

Another possibility is that the range of local compositions arises from concentration fluctuations. Concentration fluctuations should become important over volumes greater than the Kuhn length. For PEO at $353 \mathrm{~K}$, the Kuhn length is $8 \AA,{ }^{27}$ and the $Q$ that defines a region of this size (sphere of radius $4 \AA$ )is $\sim 1.6 \AA^{-1}$. This corresponds reasonably well to the spatial region where the influence of local environments becomes evident. In the concentration fluctuation theory, the cooperative volume of a given local composition controls its mobility. The cooperative volumes are expected to diverge with temperature-specifically it is predicted that the cooperative volume of the high $T_{\mathrm{g}}$ component will diverge below that component's $T_{\mathrm{g}}{ }^{28}$ Below this temperature, local compositions defined over a small volume may occur, but the volume is not large enough to be mobile, whereas other larger volumes of the same local composition could be mobile. Thus a small volume of $\phi_{\mathrm{i}, \mathrm{PEO}}$ rich in PMMA would only be mobile if it were a part of a larger volume with the same local composition. Our data are consistent with this idea-provided that the cooperative volumes associated with the PMMA-rich side of the $P\left(\phi_{\mathrm{PEO}}\right)$ are greater than $6 \AA$ but less than $22 \AA$. The cooperative length scale for each component in several miscible blends has been extracted from available data based on the average effective composition. ${ }^{28}$ For the low $T_{\mathrm{g}}$ component, such as $\mathrm{PEO}$ in the PEO/ PMMA blend, cooperative volumes are insensitive to temperature and composition, and range from 0.4 to 0.8 times the Kuhn length. It is thus possible that with decreasing temperature the spatial scale of the measurement could become smaller than the cooperative volume. Our results could also indicate that, as expected, the influence of concentration fluctuations emerges when local compositions are defined over length scales greater than the Kuhn length.

It is clear from the data presented here that the distribution of local compositions surrounding PEO protons influences their mobility. The $Q$ dependence of the stretching parameter (Fig. 4) is not observed in QENS measurements on pure polymers (see pure PEO in Fig. 4), nor was it found for the high $T_{\mathrm{g}}$ component in this blend, PMMA. ${ }^{7}$ The effect is also present in the average relaxation times: in Figure 6 relaxation times at small spatial scales are smaller than expected, and in Figure 7, the scaling of relaxation times with spatial scale becomes greater at small spatial scales. Since local compositions further from the bulk composition are expected at small spatial scales, these observations are consistent with a distribution centered around a larger $\phi_{\mathrm{PEO}}$.

It appears contradictory that the present results are consistent with the idea of concentration fluctuations, whereas those of Lutz et al. ${ }^{6}$ suggest they are relatively unimportant. One aspect to consider in this regard is that the two studies actually examine two different blends: Lutz et al. ${ }^{6}$ examined dPEO/hPMMA and the current investigation examines hPEO/dPMMA. Because the $\chi$ parameter is small for this blend, it is likely that this "swap effect" 29 is important here. One of these blends could be much closer to a phase boundary than the other, and thus have differing levels of concentration fluctuations. This must also be considered when examining the results of García Sakai et al., ${ }^{7}$ also on a dPEO/hPMMA blend, where it appears the distribution of local compositions has little effect on PMMA dynamics. The swap effect in PEO/ PMMA has been studied with SANS $^{30}$ in the temperature range of $423-473 \mathrm{~K}$ with volume fractions of PEO from 0.10 to 0.27 . The swap effect is evident, with the system shifted away from a phase boundary for the dPEO/hPMMA blend at $423 \mathrm{~K}$. As concentration fluctuations will 
be more pronounced near a phase boundary, this is consistent with present observations $(\mathrm{hPEO} /$ dPMMA appears more influenced by concentration fluctuations), and suggests that the QENS results following PMMA mobility in dPEO/ hPMMA should be taken together with the NMR results following PEO mobility in the same system. Two additional factors suggest a difference between the two systems: the composition dependence is not the same, and the hPEO/dPMMA system is more stretched. For the dPEO/hPMMA system, very little composition dependence of relaxation times is observed, whereas in the current results the $10 \%$ blend is significantly different from the others (see Fig. 1). The stretching parameters for the dPEO/hPMMA system are $\sim 0.27$, whereas the present results find that at high temperature, $\beta$ is typically around 0.18 . This difference is small, but may suggest a wider distribution of local environments in the latter case, again consistent with a more prominent role of concentration fluctuations in hPEO/dPMMA.

Finally, we consider the chain connectivity model. In this case, the relevant length scale is temperature and composition independent, and equal to a Kuhn length. As mentioned above, this model describes the temperature dependence of the average relaxation time, and a distribution of times does not enter explicitly into the theory. To predict $\tau(T)$, VTF parameters for the pure component of interest are required. Since we have measured pure PEO for only one temperature just above the melting point, this is not possible. We used the parameters obtained earlier (Lutz et al. ${ }^{6}$ ) for dPEO to predict $\tau(T)$ for the blend for all spatial scales. A reasonable fit can only be obtained over a narrow spatial range: $1.1 \AA^{-1}$ $<Q<1.5 \AA^{-1}$, for the entire range of temperatures measured. At other spatial scales, fits cannot be obtained with any value of $\phi_{\text {self. }}$. It is notable that this region covers local compositions that are defined over spatial scales just above the Kuhn length for PEO (8-11 $\AA$ ). Within the chain connectivity model, a large variety of local compositions can exist and give rise to extremely stretched relaxations, as observed here. It is also possible that those local compositions rich in PMMA move outside the time window of the spectrometer as temperature is lowered. This would result in an averaging that incorporates progressively more $\mathrm{PEO}$ rich regions with decreasing temperature, such that the average relaxation time is faster than expected. The temperature dependence of relaxation times would thus be flatter than anticipated by the theory, which would thus not describe the data well at small spatial scales. Both these suggestions are supported by our data.

\section{CONCLUSION}

We have investigated the impact of a distribution of local compositions on the dynamics of hPEO in dPMMA. This distribution is an integral part of the concentration fluctuation theory of blend dynamics. The dynamic response of this blend is extremely stretched. This establishes the existence of a wide distribution of relaxation times, but does not by itself indicate a wide range of local compositions. To make this connection, we eliminate local compositions rich in PMMA from consideration by reducing the spatial scale to values approaching the closest intermolecular spacing. In this situation, the distribution narrows considerably-approaching the width of pure PEO. This means that the extreme dynamic heterogeneity in dynamics of hPEO in dPMMA is linked to the local compositions, rather than originating from fast and slow regions narrowly distributed in composition.

Financial support for this work was provided by the National Science Foundation, Polymers Program, through a CAREER grant DMR-0134910. This work used facilities supported in part by the National Science Foundation under Agreement No. DMR-0086210.

\section{REFERENCES AND NOTES}

1. Lodge, T. P.; McLeish, T. C. B. Macromolecules 2000, 33, 5278 .

2. He, Y.; Lutz, T. R.; Ediger, M. D. J Chem Phys 2003, 119, 9956.

3. Zetsche, A.; Fischer, E. W. Acta Polym 1994, 45, 168.

4. Katana, G.; Fischer, E. W.; Hack, T.; Abetz, V.; Kremer, F. Macromolecules 1995, 28, 2714.

5. Kumar, S. K.; Colby, R. H.; Anastiasadis, S. H.; Fytas, G. J Chem Phys 1996, 105, 3777.

6. Lutz, T. R.; He, Y.; Ediger, M. D.; Cao, H.; Lin, G.; Jones, A. A. Macromolecules 2003, 36, 1724.

7. García Sakai, V.; Chen, C.; Maranas, J. K.; Chowdhuri, Z. Macromolecules 2004, 37, 9975.

8. Copley, J. R. D.; Cook, J. C. Chem Phys 2003, 292, 477.

9. Meyer, A.; Dimeo, R. M.; Gehring, P. M.; Neumann, D. A. Rev Sci Instrum 2003, 74, 2759.

10. The IDL-based DAVE program can be found at http://www.ncnr.nist.gov/dave/. 
11. Zorn, R.; Arbe, A.; Colmenero, J.; Frick, B.; Richter, D.; Buchenau, U. Phys Rev E 1995, 52, 781.

12. Triolo, A.; Lechner, R. E.; Desmedt, A.; Telling, M. T. F.; Arrighi, V. Macromolecules 2002, 35, 7039.

13. Arialdi, G.; Ryckaert, J. P.; Theodoru, D. N. Chem Phys 2003, 292, 371.

14. Neelakantan, A.; Maranas, J. K. J Chem Phys 2004, 120, 465 .

15. Williams, G.; Watts, D. C. Trans Faraday Soc 1970, 66, 80 .

16. Colmenero, J.; Arbe, A.; Alegria, A.; Monkenbusch, M.; Richter, D. J Phys Condens Matter 1999, 11, A363.

17. Arbe, A.; Colmenero, J.; Monkenbusch, M.; Richter, D. Phys Rev Lett 1998, 81, 590.

18. Deschenes, L. A.; Vanden Bout, D. A. Science 2001, 292, 255.

19. Donati, C.; Glotzer, S. C.; Poole, P. H.; Kob, W.; Plimpton, S. J. Phys Rev E 1999, 60, 3107.

20. Farago, B.; Chen, C.; Maranas, J. K.; Kamath, S.; Colby, R. H.; Pasquale, A. J.; Long, T. E. Phys Rev $\mathrm{E}$, in press.
21. Chen, C.; Neelakantan, A.; Garcia Sakai, V.; Maranas, J. K.; Lynn, J. W. In preparation.

22. Moreno, A. J.; Alegría, A.; Colmenero, J.; Frick, B. J Chem Phys 2001, 34, 4886.

23. Arrighi, V.; Higgins, J. S.; Burgess, A. N.; Howells, W. S. Macromolecules 1995, 28, 2745.

24. Bée, M. Quasielastic Neutron Scattering; Adam Hilger: Philadelpha, 1988.

25. Jin, X.; Zhang, S.; Runt, J. Polymer 2002, 43, 6247.

26. Jin, X.; Zhang, S.; Runt, J. Macromolecules 2004, 37,8110 .

27. Graessley, W. W. Polymeric Liquids and Networks: Structure and Properties; Taylor \& Francis: London, 2004.

28. Kant, R.; Kumar, S. K.; Colby, R. H. Macromolecules 2003, 36, 10087.

29. Graessley, W. W.; Krishnamoorti, R.; Balsara, N. P.; Fetters, L. J.; Lohse, D. L.; Schultz, D. N.; Sissano, J. A. Macromolecules 1993, 26, 1137.

30. Hopkinson, I.; Kiff, F. T.; Richards, R. W.; King, S. M.; Farren, T. Polymer 1995, 36, 3523. 